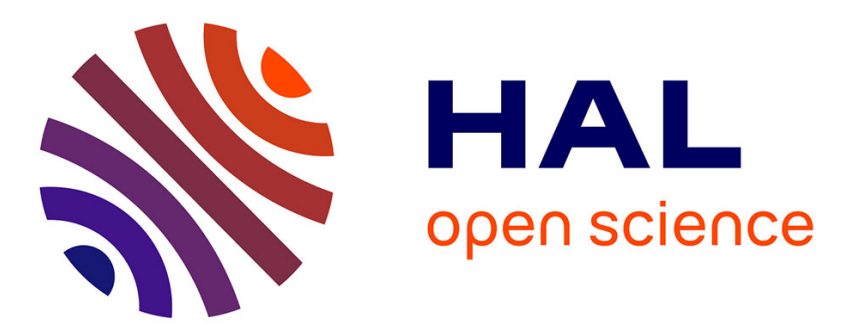

\title{
Effet des sollicitations thermomécaniques sur les transformations de phases dans l'état solide. Aspects métallurgique et mécanique
}

\author{
A. Simon, S. Denis, E. Gautier
}

\section{- To cite this version:}

A. Simon, S. Denis, E. Gautier. Effet des sollicitations thermomécaniques sur les transformations de phases dans l'état solide. Aspects métallurgique et mécanique. Journal de Physique IV Proceedings, 1994, 04 (C3), pp.C3-199-C3-213. 10.1051/jp4:1994328 • jpa-00252525

HAL Id: jpa-00252525

https://hal.science/jpa-00252525

Submitted on 1 Jan 1994

HAL is a multi-disciplinary open access archive for the deposit and dissemination of scientific research documents, whether they are published or not. The documents may come from teaching and research institutions in France or abroad, or from public or private research centers.
L'archive ouverte pluridisciplinaire HAL, est destinée au dépôt et à la diffusion de documents scientifiques de niveau recherche, publiés ou non, émanant des établissements d'enseignement et de recherche français ou étrangers, des laboratoires publics ou privés. 


\title{
Effet des sollicitations thermomécaniques sur les transformations de phases dans l'état solide. Aspects métallurgique et mécanique
}

\author{
A. SIMON, S. DENIS et E. GAUTIER
}

Laboratoire de Science et Génie des Matériaux Métalliques, LSG2M, URA 159, Ecole des Mines, parc de Saurupt, 54042 Nancy cedex, France

\begin{abstract}
When stresses are applied to a material in a metastable crystallographic structure, different effects are observed. Phase transformation kinetics is affected, a transformation plasticity deformation occurs, and transformation mechanisms can be modified.

These effects are reviewed for several phase transformations which occur in steels, and different thermomechanical paths. The interactions between stress and phase transformations are specially analysed for the martensitic transformation of ferrous alloys.
\end{abstract}

\section{Résumé}

Les contraintes mécaniques appliquées à un matériau dont la structure cristallographique est métastable provoquent différents effets. La cinétique de transformation de phases du matériau est affectée, il apparaît une plasticité de transformation et le mécanisme de transformation peut être modifié.

Ces effets sont exposés pour différentes transformations de phases qui se produisent dans les aciers et pour différents types de sollicitations thermomécaniques. Ces interactions contrainte-transformation sont plus spécialement analysées dans le cas de la transformation martensitique des alliages ferreux.

Les sollicitations thermomécaniques appliquées à un matériau qui subira des transformations de phases se rencontrent dans deux situations bien différentes. La première consiste en une déformation appliquée à une phase stable (généralement à haute température) et un refroidissement ultérieur du matériau qui amène celui-ci dans un domaine ou la phase de haute température n'est plus stable et se transforme. Cette situation a été très largement étudiée. Elle est rencontrée dans la plupart des opérations de mise en forme à chaud (forgeage, laminage à chaud...). Elle porte en général sur l'effet des grandes déformations. Nous n'en parlerons pas ici.

La deuxième consiste à appliquer une déformation ou une contrainte sur le matériau métastable. Cette déformation ou contrainte va provoquer la transformation de phases. Cette situation se rencontre lors de la mise en forme de matériau métastable (ex : cas des alliages à mémoire de forme, aciers TRIP...) ou lors du traitement thermique des matériaux. Dans ce 
dernier cas la contrainte est provoquée par les différences de température et de microstructure dans la pièce traitée (contraintes internes). Nous allons exposer ces effets.

Lorsqu'une transformation de phase s'effectue sous contrainte, il se produit deux types de modification du comportement du métal, d'une part un effet de la contrainte sur la cinétique de la transformation : c'est l'interaction que nous appellerons "métallurgique" et d'autre part une plasticité de transformation : c'est l'interaction que nous appellerons "mécanique". Il s'agit ici d'une déformation plastique qui apparaît en cours de transformation, même pour les contraintes inférieures à la limite d'élasticité du métal. Nous allons analyser successivement chacune de ces interactions en rappelant pour chacune d'elles les principaux phénomènes (1) (2) et en nous appuyant sur des études menées sur les aciers.

\section{I - INTERACTION METALLURGIQUE}

Les études ont été effectuées essentiellement pour deux types de sollicitation mécanique du matériau : des contraintes hydrostatiques (pression) et des contraintes monoaxiales (traction, compression).

\section{. Cas des contraintes hydrostatiques}

Les études montrent un retard de la transformation sous pression hydrostatique : les températures $\mathrm{A}_{3}$ et $\mathrm{A}_{1}$ du diagramme d'équilibre sont modifiées et les cinétiques de transformation sont plus lentes.

- Pour la transformation martensitique, la température Ms diminue lorsque la pression hydrostatique augmente (figure 1).

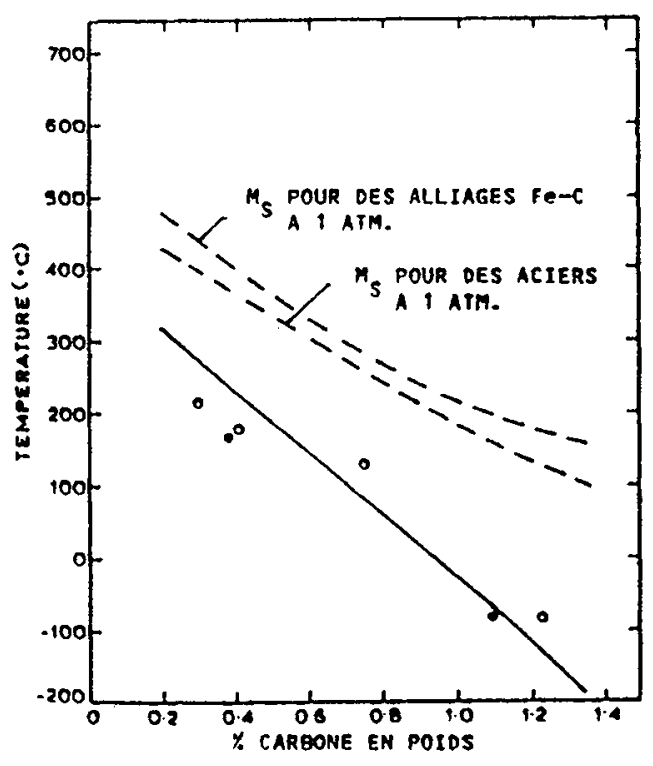

Figure 1

Influence de la pression sur la température $M_{S}$ d'alliages $F e-C$ (d'après RADCLIFFE et SCHATZ (3)) ---- $M_{S}$ calculée à $4200 \mathrm{MPa}$

O $\mathrm{MS}_{\mathrm{S}}$ mesurée à $4200 \mathrm{MPa}$ pour des alliages $\mathrm{Fe}-\mathrm{C}$

$\mathrm{M}_{S}$ mesurée à $4200 \mathrm{MPa}$ pour des aciers. 
- Pour les transformations avec diffusion, les études de cinétique de décomposition de l'austénite montrent un décalage des courbes TTT et TRC vers des températures plus basses et des temps plus longs (figure 2).

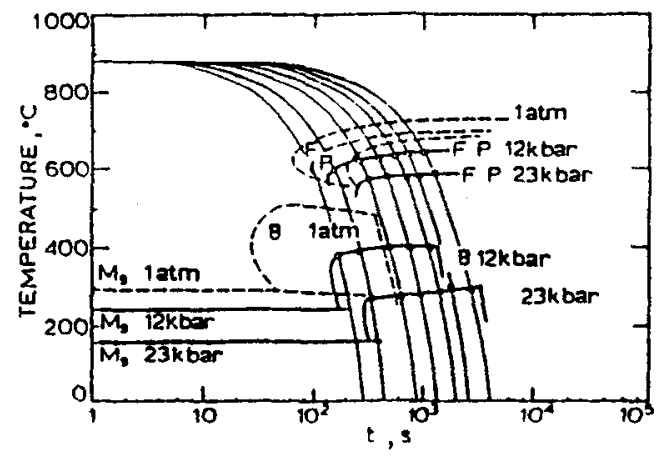

\author{
Figure 2 \\ Diagramme TRC (alliage $50 \mathrm{Cr}-4 \mathrm{~V}$ \\ austénitisé $5 m n$ à $880^{\circ} \mathrm{C}$ ) pour \\ différentes pressions (d'après \\ SCHMIDTMANN (4)).
}

\title{
. Cas des contraintes monoaxiales
}

Les modifications des cinétiques de réaction ont été étudiées pour différentes sollicitations du matériau :

- application d'une contrainte constante au cours de la transformation

- déformation variable au cours de la transformation

- déformation plastique préalable de l'austénite.

- Pour la transformation martensitique et pour le premier type d'essai (contrainte constante et température variable) le résultat principal est une augmentation de la température Ms aussi bien sous contraintes de traction que sous contraintes de compression. Cette augmentation est le plus souvent donnée comme linéaire avec la contrainte appliquée. Une contrainte de compression a un effet plus faible qu'une contrainte de traction. Un exemple de ces variations est donné sur la figure 3. Pour le deuxième type d'essai (température constante et contrainte variable), on étudie la contrainte critique pour provoquer la transformation martensitique et les cinétiques de transformation en fonction de la contrainte ou de la déformation. Les résultats obtenus peuvent le plus souvent être schématisés comme sur la figure 4 .

Dans le domaine I, la contrainte nécessaire pour induire la transformation est inférieure à la limite d'élasticité de la phase mère et crôit linéairement avec la température d'essai.

Dans le domaine II, la transformation martensitique est précédée d'une déformation plastique de la phase mère. Dans ce cas, la contrainte critique est d'abord proche de la limite d'élasticité de l'austénite puis augmente plus rapidement quand la température d'essai augmente.

En ce qui concerne les cinétiques de transformation en fonction de la contrainte appliquée ou de la déformation un exemple de résultat expérimental que nous avons obtenu (1) est donné sur la figure 5. On peut noter que l'échantillon se transforme très peu dans le domaine des contraintes élastiques $(\sigma<200 \mathrm{MPa})$, par contre, dans le domaine plastique le 
taux de martensite formée augmente rapidement avec la contrainte (figure 5a) ou la déformation (figure $5 \mathbf{b}$ ).
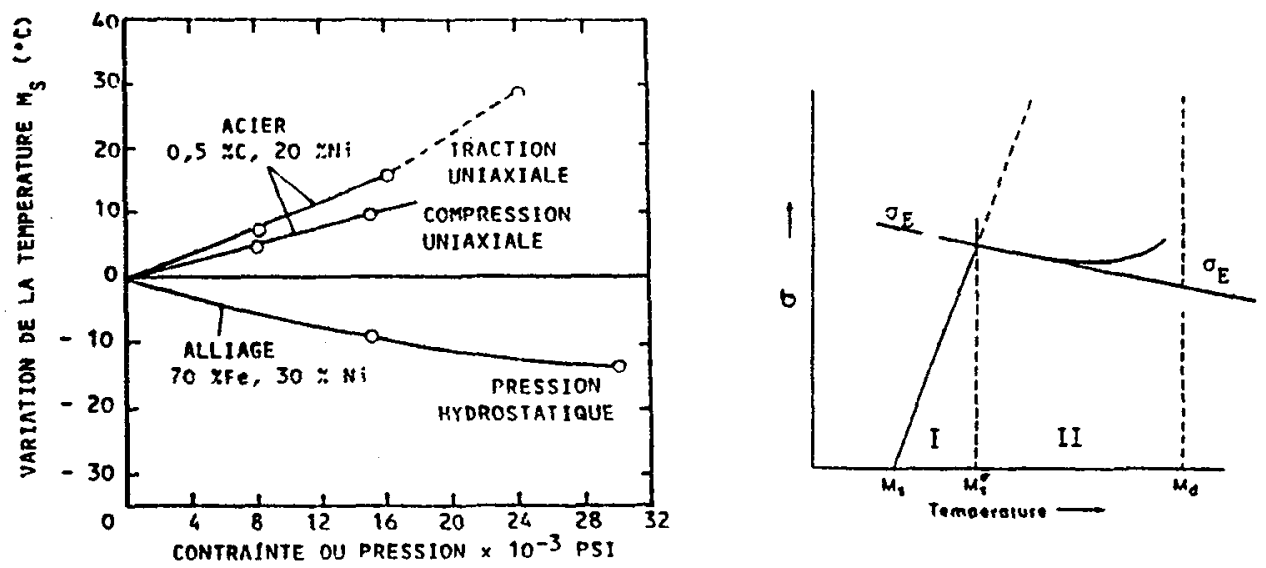

Figure 3 - Variations de la température $M_{S}$ en fonction d'une contrainte uniaxiale de traction, de compression et d'une pression hydrostatique (d'après PATEL et COHEN (5) ). $\left(1 \mathrm{psi}=6,89 \times 10^{3} \mathrm{~Pa}\right)$

Figure 4 - Représentation schématique des variations de la contrainte nécessaire pour provoquer la transformation martensitique en fonction de la température d'essai (d'après OLSON et COHEN tiré de (1)).

( $\sigma E$ limite d'élasticité de l'austénite).
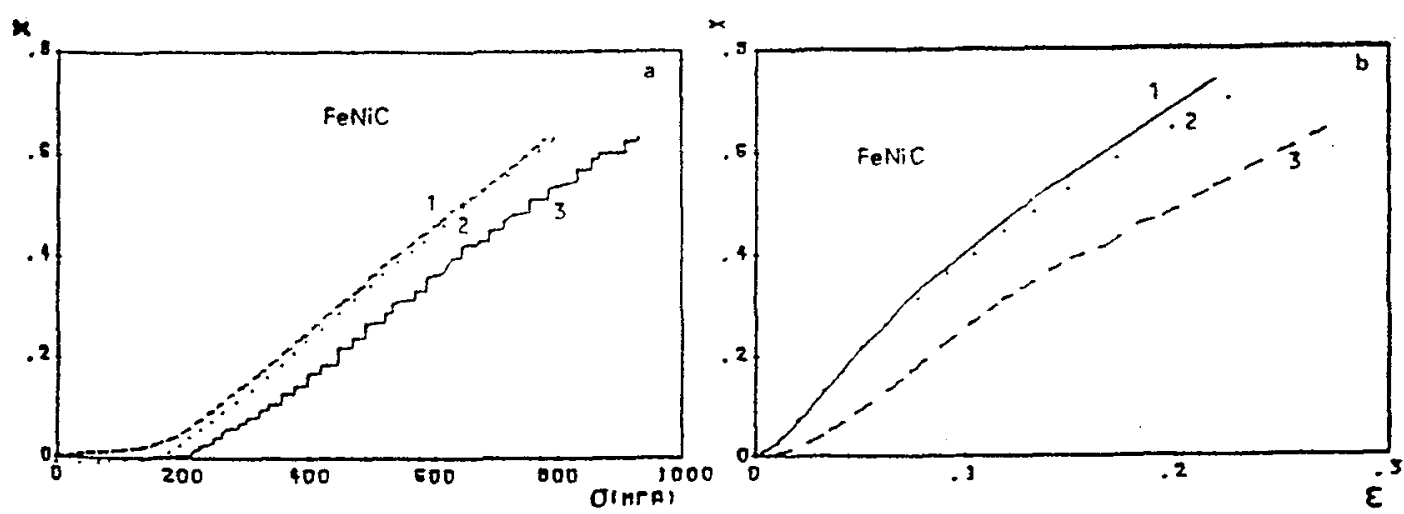

Figure 5 - a) Variation de la cinétique de transformation en fonction de la contrainte appliquée de traction ( $x$ : taux de martensite formée).

b) Variation de la cinétique de transformation en fonction de la déformation pour trois conditions de sollicitation. Alliage Fe-Ni-C. Température d'essai : $0^{\circ} \mathrm{C}$

$$
\begin{aligned}
& \text { 1. } \dot{\varepsilon}=1 \times 10^{-4} \mathrm{~s}^{-1} \\
& \text { 2. } \dot{\varepsilon}=8 \times 10^{-4} \mathrm{~s}^{-1} \\
& \text { 3. } \dot{\sigma}=2,6 \mathrm{MPa} \mathrm{s}^{-1}
\end{aligned}
$$

Le troisième type de sollicitation (déformation préalable de l'austénite) conduit aux résultats suivants : pour la plupart des auteurs une déformation plastique à haute température influence peu la température de début de transformation martensitique. Lorsque la déformation a lieu à température proche de Ms, on observe dans le cas des aciers, une 
diminution de la température Ms en fonction de la déformation (diminution d'autant plus importante que la température de déformation est proche de Ms). Par exemple, COLLETTE (6) a mesuré une diminution sensiblement linéaire de la température Ms d'un acier de type 60 NCD11 pour des prédéformations de l'austénite comprises entre 0 et $4 \%$.

$\left(\frac{\mathrm{dM}_{\mathrm{s}}}{\mathrm{d} \varepsilon}=-5^{\circ} \mathrm{C} / \%\right.$ déformation $)$

- Pour les transformations hautes températures (ferritique et perlitique) en conditions isothermes, sous contrainte constante, la cinétique de la transformation est accélérée. Les temps d'incubation et la durée de la réaction diminuent que les contraintes soient de traction ou de compression. Un exemple est donné sur la figure 6 pour la transformation perlitique sous contrainte de traction. Les études menées en terme d'analyse des vitesses de germination et des vitesses de croissance ont montré que l'accélération de la transformation est essentiellement due à une augmentation de la vitesse de germination.

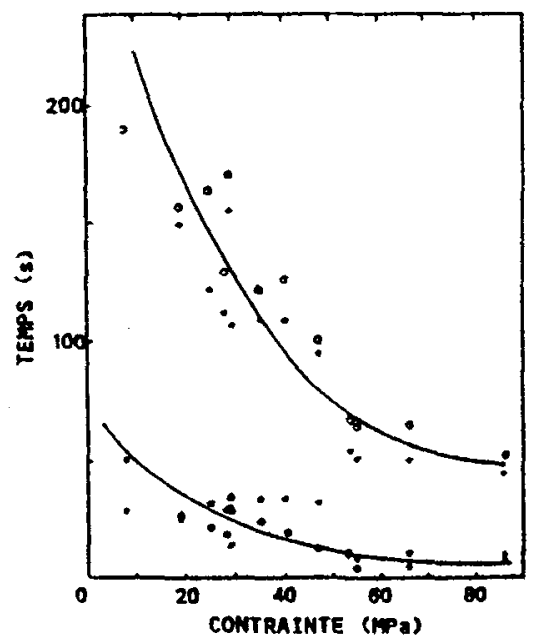

Figure 6-Variations des temps de début $\left(^{* 0}\right)$ et des temps de fin $(* 0)$ de transformation en fonction de la contrainte appliquée de traction pour la transformation perlitique isotherme $\left(\grave{a} 673^{\circ} \mathrm{C}\right)$ d'un acier XC80 (GAUTIER (1)).

Pour les autres types de sollicitation, les phénomènes observés sont les mêmes : décalage des courbes TTT ou TRC vers des temps plus courts, accélération de la cinétique de transformation.

- Pour la transformation bainitique, lorsqu'une contrainte constante est appliquée au cours de la transformation, la transformation est accélérée comme dans le cas des transformations ferritique et perlitique. Pour une déformation plastique préalable de l'austénite, les phénomènes sont moins nets : la transformation est ralentie quand la déformation a lieu à température élevée par rapport à la température de transformation et accélérée lorsque la déformation a lieu à une température proche du domaine de transformation.

\section{II - INTERACTION MECANIQUE}

Lorsqu'on applique une contrainte mécanique à un alliage métallique au cours de la transformation de phase, il se produit une déformation supérieure à celle due à la variation volumique même pour des contraintes inférieures à la limite d'élasticité. Ce phénomène de plasticité de transformation est observé aussi bien pour les transformations 
avec diffusion que pour les transformations par cisaillement. Cette plasticité de transformation est reliée à la contrainte appliquée et à l'avancement de la transformation.

L'essentiel des études a été effectué pour des contraintes appliquées monaxiales (traction ou compression), la contrainte appliquée étant constante durant la transformation. Ce sont les essais dits statiques.

Des études plus récentes (1) (7) (8) (9) concernent aussi la plasticité de transformation pour des essais dits dynamiques pour lesquels la transformation se produit au cours d'un essai de traction.

Nous nous limiterons ici aux résultats obtenus dans le cas des essais statiques.

La plupart des résultats rapportés montrent une relation linéaire de la plasticité de transformation avec la contrainte appliquée pour des contraintes inférieures à la limite d'élasticité (n'induisant pas de fluage) et pour une transformation complète. La figure 7 donne un exemple d'une telle évolution pour la transformation martensitique d'un acier de type 60 NCD11 sous contrainte de traction (COLLETTE (6)). Une évolution très similaire est obtenue par DESALOS (10) pour la transformation martensitique sous contrainte de compression d'un acier de type 35 NCD16 (figure 8).
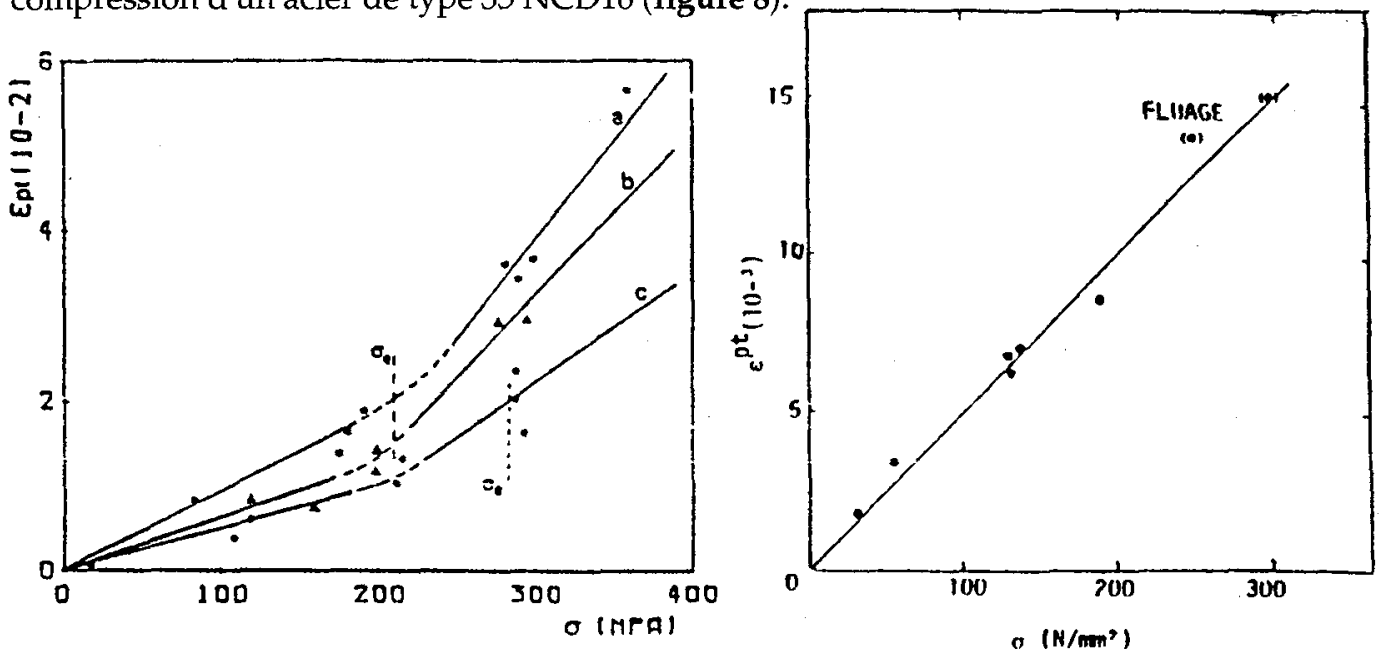

Figure 7 - Variations de la déformation de plasticité de transformation en fonction de la contrainte appliquée pour une transformation complète (tiré de (1)). Acier 60NCD11.

a: austénitisation à $1050^{\circ} \mathrm{C}-V_{R}=0,5^{\circ} \mathrm{C} / \mathrm{s}$

$\left(V_{R}\right.$ : vitesse de refroidissement dans le domaine de transformation).

$b$ : austénitisation à $1050^{\circ} \mathrm{C}-V_{R}=5^{\circ} \mathrm{C} / \mathrm{s}$

$c$ : austénitisation à $900^{\circ} \mathrm{C}$

$\sigma_{\mathcal{e}}$ limite d'élasticité de l'austénite (à tempé-

rature proche de $M_{S}$ ).
Figure 8 - Influence de la contrainte axiale appliquée (de compression) avant transformation sur la déformation de plasticité de transformation totale d'un acier 35 NCD16 (austénitisé à $900^{\circ} \mathrm{C}$ ) (d'après DESALOS (10) ). 
Il existe beaucoup moins de résultats expérimentaux reliant la plasticité de transformation et l'avancement de la transformation. Dans le cas de la transformation martensitique, suivant le type d'alliage étudié les différents auteurs observent des variations linéaires (11) ou non linéaires (7) (12) (1) de la plasticité de transformation en fonction du taux de phase transformée. Un exemple de telles variations est donné sur la figure 9 pour un alliage FeNiC. On peut noter que pour cet alliage, la variation de la plasticité de transformation est plus importante au début de la transformation et diminue ensuite. De telles évolutions sont aussi obtenues pour des alliages FeNiCCr (1) 60NCD11 (1) et 35NCD16 (10).

Nous avons étudié (1) cette plasticité de transformation pour la transformation perlitique d'un acier eutectoïde, en condition isotherme sous contrainte de traction. Les résultats obtenus sont donnés sur la figure 10.

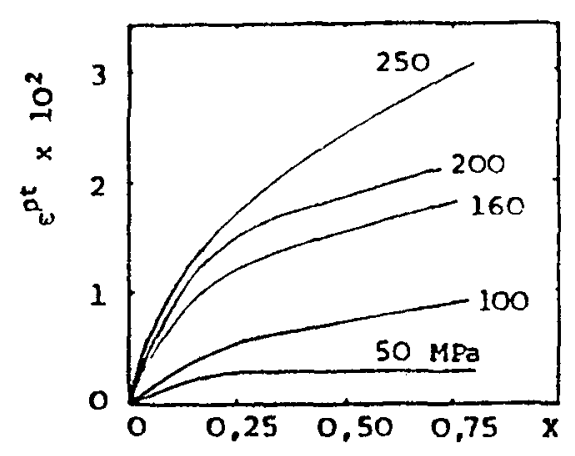

Figure 9 - Variations de la déformation de plasticité de transformation en fonction $d u$ taux de martensite formée pour différentes contraintes de traction appliquées.

Alliage Fe-Ni-C (GAUTIER (1)).

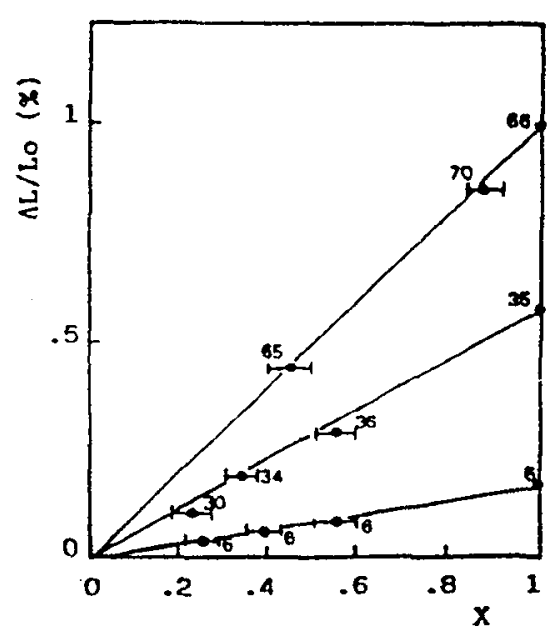

Figure 10 - Variation de l'amplitude dilatométrique de transformation en fonction $d u$ taux de perlite formée pour différentes contraintes de traction appliquées ( $6 \mathrm{MPa}, 35$ $M P a, 66 \mathrm{MPa}$ )

Acier XC 80 - Température de transformation $673^{\circ} \mathrm{C}$ (GAUTIER (1)).

\section{III - EXEMPLE DE LA TRANSFORMATION MARTENSITIOUE DES ACIERS}

C'est pour ce type de transformation que nous pouvons aborder toute la diversité des phénomènes qui interviennent lors des interactions contraintes transformations de phases.

Pour des contraintes monoaxiales, la modélisation est basée sur le concept de SCHEIL : la transformation martensitique est un mode de déformation composé d'un cisaillement et d'une dilatation (figure 11). 

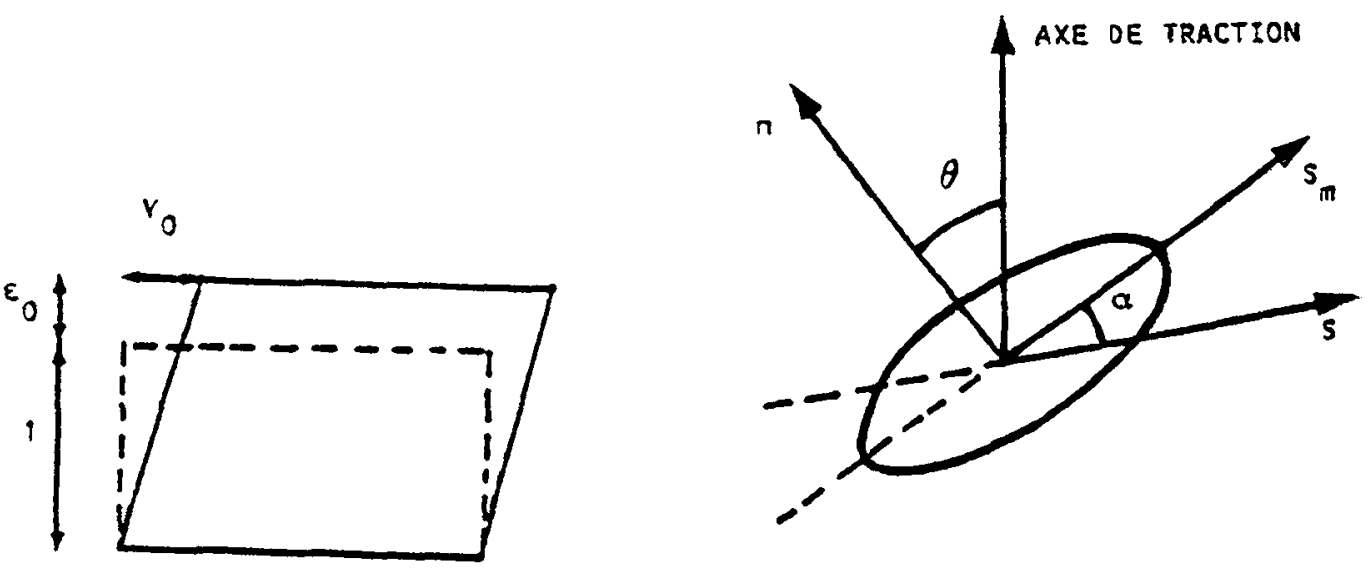

Figure 11 : Déformation liée à la transformation martensitique ( $\varepsilon_{0}$ : dilatation normale au plan d'habitat ; $\gamma_{0}$ : cisaillement parallèle au plan d'habitat; $n:$ normale au pland'habitat ; $s_{m}$ : direction de cisaillement maximum; $s$ : direction de cisaillement).

Si la transformation se produit sous contrainte, un travail peut être associé à cette déformation $W=(\underset{\sim}{\sigma} \underset{\sim}{\varepsilon}) \mathrm{Vm}$ (où $\sigma$ et $\varepsilon$ dont les tenseurs de contrainte et de déformation de transformation et Vm le volume de martensite formé).

Le bilan thermodynamique lors de la formation de la première plaquette s'écrit :

$\Delta G_{c}^{\gamma-\alpha}+\Delta G_{\text {surf }}^{\gamma-\alpha^{\prime}}+\Delta G_{D}^{\gamma-\alpha}+W=0$

$\Delta \mathrm{G}_{\mathrm{c}}^{\gamma-\alpha^{\prime}}$ : différence d'énergie libre entre la phase $\gamma$ et la phase $\alpha$

$\Delta G_{\text {surf }}^{\gamma-\alpha^{\prime}}$ : énergie interfaciale

$\Delta G_{D}^{\gamma-\alpha}:$ énergie de déformation

W : travail associé à la transformation

Patel et Cohen [5] ont exprimé ce travail par la relation suivante :

$\mathrm{W}=\mathrm{Vm}\left[\sigma \cdot \frac{1}{2}\left[\gamma_{0} \sin 2 \theta+\varepsilon_{0}(1+\cos 2 \theta)\right]\right](+$ traction $;-$ compression $)$

(dans ce cas , $\alpha$, défini figure 11, est égal à 0 , la direction de cisaillement la direction de cisaillement maximum).

Une valeur, $W$ maximum peut être obtenue suivant la valeur de $\theta$, c.a.d. l'orientation de la plaquette de martensite par rapport à la contrainte appliquée pour $\frac{\sin 2 \theta}{\cos 2 \theta}= \pm \frac{\gamma_{0}}{\varepsilon_{0}}$ avec $\frac{\mathrm{dW}}{\mathrm{d} \theta}=0$ 
Cette approche conduit à un décalage de la température Ms de l'acier et à une orientation préférentielle des plaquettes de martensite par rapport à la direction de la contrainte appliquée.

\section{1 Modélisation de l'effet d'une contrainte uniaxiale sur Ms}

PATEL et COHEN (5) ont établi une relation donnant les variations de la température Ms pour des contraintes de traction, de compression ou une pression hydrostatique. Ils supposent que la contrainte appliquée constitue une contribution mécanique à la force motrice thermodynamique pour la transformation. Ainsi ils prévoient que la composante de cisaillement de la contrainte favorise la transformation alors que la composante normale la favorise si elle est de traction et s'y oppose si elle est de compression. Leur relation est la suivante :

$$
\frac{\mathrm{dMs}}{\mathrm{d} \sigma}=\frac{1}{2}\left[\gamma_{\mathrm{o}} \sin 2 \theta+\varepsilon_{\mathrm{o}}(1+\cos 2 \theta)\right] / \frac{\mathrm{d}\left(\Delta \mathrm{G}_{\mathrm{c}}^{\gamma-\alpha}\right)}{\mathrm{dT}}
$$

Le modèle prévoit que la température Ms augmente sous l'effet d'une contrainte de compression, augmente plus sous l'effet d'une contrainte de traction et diminue pour une pression hydrostatique. Ces variations sont confirmées expérimentalement et les auteurs obtiennent un bon accord calcul-expérience pour des alliages Fe-Ni et Fe-Ni-C. Néanmoins l'application de cette relation à d'autres alliages nécessite une bonne connaissance de la transformation sous contrainte (en particulier les valeurs de $\gamma_{0}$ et $\varepsilon_{0}$ tirées de la littérature ne permettent pas d'aboutir à des prévisions quantitatives en accord avec l'expérience dans le cas d'aciers d'utilisation courante). Pour un acier de type $60 \mathrm{NCD} 11, \frac{\mathrm{dMs}}{\mathrm{d} \sigma}$ calculé $=0,12^{\circ} \mathrm{C} / \mathrm{MPa}$, $\frac{\mathrm{dMs}}{\mathrm{d} \sigma}$ expérimental $=0,05^{\circ} \mathrm{C} / \mathrm{MPa}(1)$.

Notons que ce modèle concerne des contraintes appliquées dans le domaine élastique.

\section{2 Plasticité de transformation}

La plasticité de transformation pour la transformation martensitique est attribuée à deux mécanismes principaux schématisés dans la figure $\mathbf{1 2}$.

1) Orientation des plaquettes de martensite par la contrainte appliquée. La déformation résultante est réversible lorsque la martensite se retransforme en austénite. Cet effet est dû à la composante de cisaillement qui se produit durant la transformation de phases. Ce mécanisme est illustré par la superélasticité et l'effet mémoire de forme dans les alliages Cu$\mathrm{Zn}-\mathrm{Al}$ et dans d'autres alliages.

2) Orientation de la déformation plastique qui se produit autour des zones qui se transforment. La déformation de transformation martensitique est accommodée par la déformation plastique de l'austénite. Ce mécanisme a été largement étudié pour les transformations avec diffusion $(13,14,2)$. 

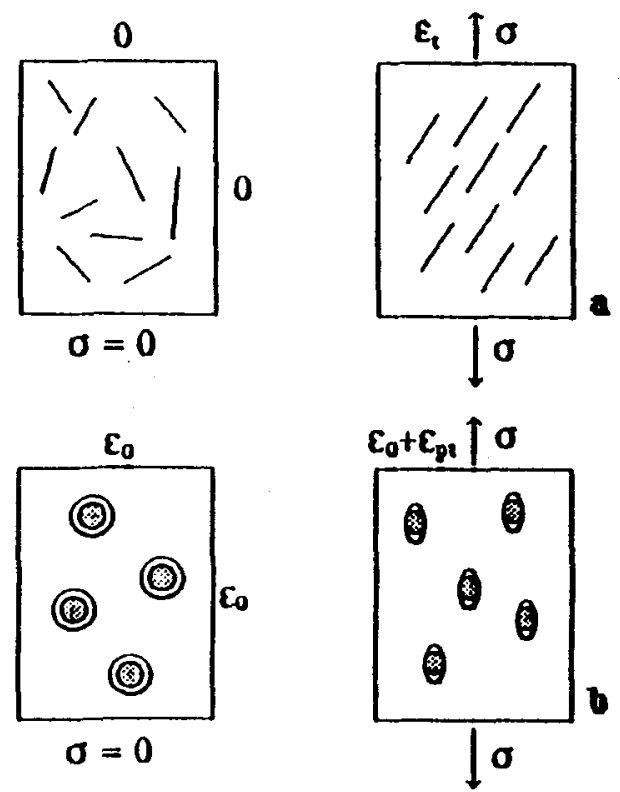

Figure 12 : Représentation schématique de la plasticité de transformation.

a: sélection de l'orientation des plaquettes de martensites (12)

$b$ : accommodation anisotrope de la déformation de transformation ( $\Delta V / V$ dans ce cas) au cours de la transformation (13).

Ces deux mécanismes sont actifs dans le cas de la transformation martensitique des alliages ferreux car celle-ci met en jeu une déformation de cisaillement macroscopique importante $(\simeq 0,2)$ et une variation volumique $(\simeq 0,03)$.

Nous avons étudié la plasticité de transformation en fonction de la progression de la transformation pour des alliages Fe-20 Ni-0,5C $\left(\mathrm{Ms}=-7\right.$ ou $\left.-16^{\circ} \mathrm{C}\right)$. Différents essais ont été réalisés :

- des essais de fluage anisotherme (essais "statiques"), l'échantillon est refroidi sous contrainte constante depuis la température ambiante jusqu'à une température inférieure à la température Ms ;

- des essais de traction (essais "dynamiques"), l'échantillon est déformé en traction avec une vitesse de mise en charge constante $\left(1,8 \mathrm{Ns}^{-1}\right)$ à température constante, comprise entre Ms et Md.

Ces résultats expérimentaux ont été décrits et analysés (16) ; les principales conclusions sont les suivantes :

- pour les essais de fluage anisotherme, l'orientation favorable des plaquettes de martensite par la contrainte appliquée se produit au début de la transformation (jusqu'à $10 \%$ de transformation) et pour des contraintes qui atteignent la limite d'élasticité de l'austénite. L'accommodation plastique anisotrope des déformations de transformation se produit également, mais son effet doit être faible ( $\simeq 1$ à $2 \%$ ). 
- pour les essais de traction, l'orientation des plaquettes de martensite se produit mais essentiellement dans le domaine de déformation plastique. Pour ces essais nous observons des déformations de plasticité de transformation importantes. Cette déformation de transformation "normalisée" par la progression de la transformation $\left(\frac{\mathrm{d} \varepsilon_{\mathrm{pt}}}{\mathrm{dx}}\right.$, avec $\mathrm{d} \varepsilon_{\mathrm{pt}}=$ incrément de plasticité de transformation et $\mathrm{dx}$ progression de la trans-formation) atteint une valeur comprise entre 0,4 et 0,6 . Dans des conditions thermomécaniques similaires (contrainte-température) la valeur maximale de la plasticité de transformation est de 0,12 pour un essai à contrainte appliquée constante.

Les valeurs élevées de plasticité de transformation $(0,4$ à 0,6$)$ ne peuvent s'expliquer uniquement par une orientation complète des plaquettes de martensite.

En effet la déformation associée à la transformation dans la direction de la contrainte appliquée est : $\varepsilon_{\mathrm{t}}=\frac{1}{2}\left(\gamma_{\mathrm{o}} \sin 2 \theta \cos \propto+\varepsilon_{\mathrm{o}}(1+\cos 2 \theta)\right) \quad$ (5) et figure 11 .

Cette déformation dépend de l'orientation de la plaquette de martensite par rapport à la direction de la contrainte appliquée. Sa valeur maximale sera obtenue pour $\operatorname{tg} 2 \theta=\gamma_{0} / \varepsilon_{0}$. Dans le cas de l'alliage Fe-Ni-C $\varepsilon_{\text {tmax }}=0,11$ avec $\gamma_{\mathrm{o}}=0,19$ et $\varepsilon_{\mathrm{o}}=0,024$

Pour un monocristal et dans le cas d'une variation volumique durant la transformation négligeable, il y a simplement orientation de la martensite. Dans un polycristal la plasticité de transformation sera une fonction de la distribution de l'orientation des plaquettes. Cette orientation des plaquettes sera dépendante de la contrainte appliquée $\sigma_{\mathrm{A}}$ et interne $(\sigma \mathrm{I})$ générées lors de la transformation, (interactions entre les plaquettes, entre les grains). Ainsi l'orientation des plaquettes est déterminée par la contrainte locale $\sigma=\sigma_{\mathrm{A}}+\sigma_{\mathrm{I}}$. Si $\sigma_{\mathrm{A}}$ est important par rapport à $\sigma_{\mathrm{I}}$, l'orientation des plaquettes de martensite sera déterminée essentiellement par $\sigma_{\mathrm{A}}$ et la déformation résultante sera une plasticité de transformation qui tend vers $\varepsilon_{\mathrm{t}} \max$. Si $\sigma_{\mathrm{A}}$ à une faible contribution au tenseur local, l'orientation des plaquettes sera déterminée par $\sigma_{\mathrm{I}}$ essentiellement. Alors les orientations des plaquettes seront multiples et la déformation dans la direction de la contrainte appliquée sera plus faible.

Ainsi nous prévoyons une contribution maximale de l'orientation des plaquettes de martensite lorsque $\sigma_{\mathrm{A}} \gg \sigma_{\mathrm{I}}$ c.a.d. au début de la transformation et lorsque l'accommodation des déformations de transformation est essentiellement élastoplastique (pas d'auto accommodation). Ceci se produit au début des essais de fluage anisotherme sous forte contrainte et dans le domaine de la déformation plastique en essai de traction (15).

A présent, nous développons une modélisation de ces phénomènes par une approche micromécanique en éléments finis (17). 


\section{3 Modification des mécanismes de transformation}

Précedemment, nous avons considéré que les mécanismes de formation de la plaquette de martensite ne sont pas modifiés, lorsque la transformation se produit sous contrainte appliquée. Cependant des changements peuvent être observés notamment lorsque la martensite est formée durant un essai de traction $(16,18,19)$. Les modifications importantes que nous avons observées sont montrées sur la figure 12 bis et discutées dans $(16,18)$.

Lorsque la martensite se forme au cours d'un essai de traction, pour des contraintes faibles, le plan d'accolement de la martensite est $\{225\} \gamma$ et la croissance de la plaquette est stoppée le long de \{111\} (figure 12a), la grande direction de croissance de la martensite est $\{225\} \gamma$. Ceci est identique à la formation de ce type de martensite au cours du refroidissement. Lorsque la transformation s'effectue sous contraintes plus élevées, durant la déformation plastique, les plaquettes de martensite se forment encore le long des plans $\{225\} \gamma$ mais elles présentent également des directions de croissance le long des plans $\{111\} \gamma$ (figure $\mathbf{1 2 b}$ ).

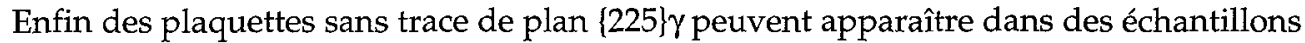
transformés à plus haute température et pour des déformations plus importantes (figure 12c).

Le changement de plan d'accolement peut être relié à une modification du mécanisme d'accommodation de la déformation de BAIN. Ainsi, TIEM (20) a calculé une modification du plan d'habitat vers $\{111\} \gamma$ lorsque la déformation de transformation (déformation de Bain) est accommodée par une déformation plastique de l'austénite et lorsqu'une contrainte de traction est appliquée.

Si la transformation se produit dans le domaine de déformation plastique de l'austénite, l'accommodation de la déformation de Bain se produit différemment (changement du maclage dans la martensite à du glissement dans la martensite et l'austénite). Une déformation de plasticité de transformation peut alors en résulter, permettant d'expliquer les valeurs élevées de plasticité obtenues au cours des essais de traction.

Nous pensons que les sollicitations thermomécaniques qui existent durant un essais de traction atteignent localement des niveaux tels que de nouveaux plans de glissement dans l'austénite deviennent actifs. Ces plans facilitent l'accommodation par glissement de la déformation de Bain.

Comme nous le montre la microstructure de la figure $\mathbf{1 2 b}$, les plaquettes se forment souvent avec un plan d'accolement $\{225\} \gamma$ et ensuite se développent le long de $\{111\} \gamma$. Le plan d'accolement initial peut être considéré comme inchangé, mais la croissance est ensuite complètement modifiée.

Pour ces essais, la contrainte a deux effets. D'une part, elle sélectionne le variant de martensite par le plan d'habitat initial, d'autre part, elle modifie le mécanisme de croissance. La croissance est gouvernée par la déformation qui provoque la morphologie décrite par la micrographie $12 \mathrm{c}$ et décrite par le schéma de la figure $13 \mathrm{c}$. 


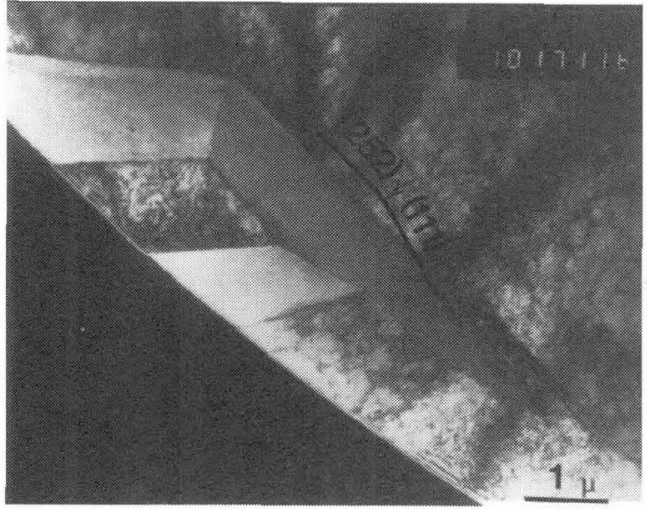

Température d'essai : $M s+8{ }^{\circ} \mathrm{C}$ $\varepsilon=6,4 \%$

a

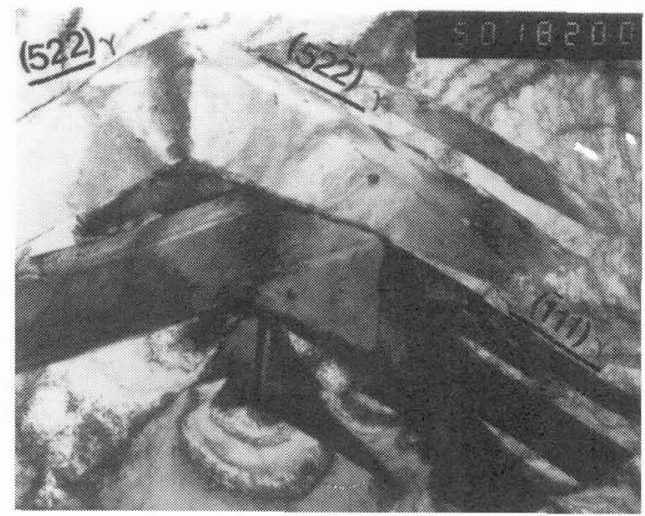

Température d'essai $\mathrm{Ms}+8^{\circ} \mathrm{C}$ $\varepsilon=6,4 \%$ (état intermédiaire entre a et $c$ )

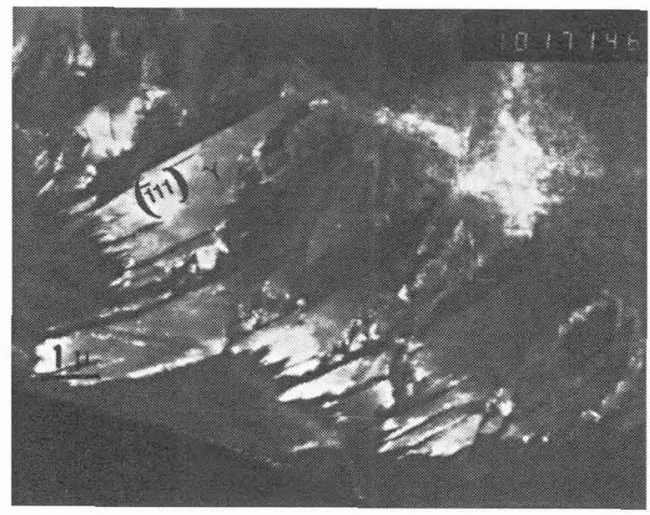

b

Température d'essai $\mathrm{Ms}+20^{\circ} \mathrm{C}$

$$
\varepsilon=12 \%
$$

Figure 12bis: Morphologies de martensites formées au cours d'essais de traction. Ces variations de morphologies sont illustrées schématiquement sur la figure 13. 


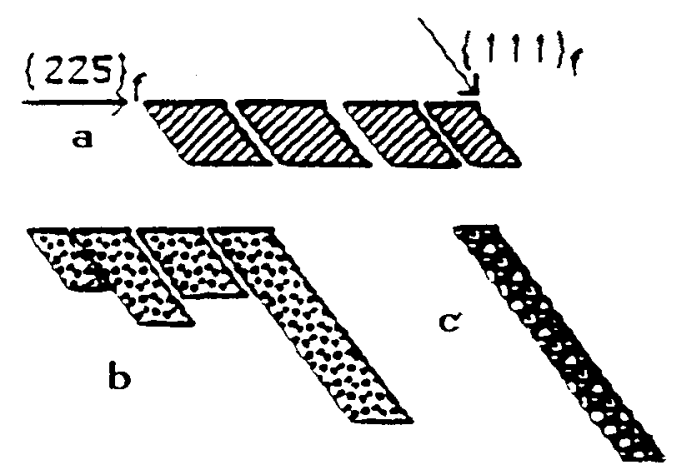

Figure 13: Schéma de variation de morphologies des plaquettes de martensite.

a) Formation autocatalytique de sous plaquettes de martensite de plan d'accolement moyen 225$\} \gamma$ bloquées le long d'interfaces $\{111\} \gamma$.

b) Formation de sous plaquettes, la croissance selon \{111\} yest favorisée pour certaines plaquettes.

c) La croissance est favorisée le long des interfaces 1111$\}$. Accommodation de la déformation de Bain par glissement dans l'austénite.

\section{CONCLUSION :}

Les interactions entre contrainte-déformation et transformation de phases conduisent à un comportement thermomécanique très complexe du matériau qui subit une transformation de phase tout en étant sollicité mécaniquement.

Les approches qui ont été menées sont très diverses :

- au niveau des types de transformation : avec changement de densité et/ou avec cisaillement, avec germination et croissance par diffusion,

- au niveau du type de sollicitation thermomécanique :

* température constante - contrainte variable

* contrainte constante - température variable

pour des états de contraintes monoaxiaux (traction, compression) et triaxiaux (pression hydrostatique)

- au niveau du type de l'échelle de modélisation :

* approche théorique (thermodynamique, théorie des dislocations...)

* approche phénoménologique (équations de lois d'évolution à partir d'expériences).

L'ensemble de ces interactions est très complexe, et il n'est actuellement pas possible de retenir une modélisation fondamentale et universelle du comportement thermomécanique d'un matériau qui subit une transformation de phase et qui prend en compte l'ensemble des phénomènes. Néanmoins des modélisations phénoménologiques nous ont permis de prendre en compte des interactions contraintes transformations de phases dans le calcul des traitements thermiques $(21,29)$. 


\section{REFERENCES}

1- E. GAUTIER

Thèse de Doctorat ès-Sciences Physiques, Institut National Polytechnique de Lorraine, Nancy (1985).

2- E. GAUTIER, A. SIMON et G. BECK Acta Metall. 35, n6 (1987) 1367.

3- S.V. RADCLIFFE and M. SCHATZ

Acta Metall. 10 (1962) 201.

4- E. SCHMIDTMANN, H. GRAVE and F.S. CHEN

Trait. Therm., 115 (1977) 57.

5- J.R. PATEL and M. COHEN

Acta Metall. 1 (1953) 531.

6- G. COLLETTE

Thèse de Docteur-Ingénieur, Institut National Polytechnique de Lorraine, Nancy (1980).

7- F. ABRASSART

Thèse de Doctorat ès-Sciences Physiques, Université de Nancy I (1972).

8- T. NARUTANI, G.B. OLSON and M. COHEN

Proc. Int. Conf. on Martensitic Transformations (ICOMAT 1982), Ed. L. DELAEY, M. CHANDRASEKARAN, LEUVEN (Belgium) (1982).

9- C. LIEBAUT, E. GAUTIER, A. SIMON

Mem. Sci. Rev. Metall. 85 (1988) 571.

10- Y. DESALOS, R. LAURENT, J. GIUSTI and D. RADENKOVIC IRSID, ELF AQUITAINE, ECOLE POLYTECHNIQUE, Rapport Annuel, D.G.R.S.T. (1980).

11- J.F. DELORME

Thèse de Doctorat ès-Sciences Physiques, Université Claude Bernard, Lyon (1971).

12- C.L. MAGEE

Ph. D. Thesis, Carnegie Mellon University, Pittsburg, Pa, (1966).

13- G.W. GREENWOOD, R.H. JOHNSON

Proc. Roy. Soc. 283 A (1965) 403.

14- M. DE JONG, G W RATHENAU

Acta Metall 7 (1959) 246 ; Acta Metall 9 (1961) 714.

15- E. GAUTIER, A. SIMON, G. BECK

Proc. 7th Int. Conf. on Strengh of Metals and Alloys, Montreal, Canada, Pergamon Press, (1985) 569 ; Proc. ICOMAT the Japan Inst. Metals (1986) 503 ; Proc. Int. Conf. Solid-Solid Phase Transformations, Institute of Metals (1987) 285.

16- E. GAUTIER, X.M. ZHANG, A. SIMON

Proc I CRS2 Ed. G. BECK, S. DENIS, A. SIMON, Elsevier Applied Science (1989) 777.

17- J.F. GANGHOFFER, K. SIMONSSON, S. DENIS, E. GAUTIER, A. SIMON et S. SJÖSTRÖM

International Conference on Martensitic Transformation Monterey (U.S.A.) juillet 92 sous presse.

18- X.M. ZHANG, E. GAUTIER et A. SIMON

Acta Metall. 37 (1989) 477 ; Acta Metall. 37 (1989) 487

19- M. N. PANKOVA, A.L. ROYTBURD ; Phys. Met. Metall. 58 (1984) 81.

A.L. ROYTBURD, M.N. PANKOVA ; Phys. Met. Metall. 59 (1985) 131.

20- S. TIEM

Thèse de Doctorat, Université de Metz 1987.

21- S. DENIS, S. SJÖSTRÖM, A. SIMON

Metall Trans Vol 18A (1987) 1203.

22- S. DENIS, E. GAUTIER, S. SJÖSTRÖM, A. SIMON

Acta Metall 35 (1987) 1621. 